\title{
EI screencast como herramienta para desarrollar responsabilidad ética y otras competencias transversales: percepción del alumnado
}

\author{
Jaime Cebolla-Cornejo $^{\mathrm{a}}$, Miguel Leiva-Brondo ${ }^{\mathrm{b}}$, Rosa Peiróc, Ana Pérez-de-Castro ${ }^{\mathrm{d}}$
}

ajaicecor@btc.upv.es, Departamento de Biotecnología, b Departamento de Biotecnología ${ }^{c}$ ropeibar@btc.upv.es, Departamento de Biotecnología, danpede1@btc.upv.es, Departamento de Biotecnología, Universitat Politècnica de València.

\begin{abstract}
The development of amateur documentaries, in the form of screencasts, and its self-evaluation, co-evaluation and peer evaluation has proven to be a very useful instructional tool to train ethical responsibility in undergraduate students of life sciences. Part of the success is undoubtedly due to the fact of being able to take advantage of the motivation of the students, who recognize that it is an attractive challenge and that, beyond the academic assignment, they have had a good time in their development. The use of surveys to the students has allowed to verify the valoration that they make of the repercussion of the activity on the training of specific components of the ethical responsibility. In the same way, it has allowed to verify the positive effects of the tool to train other soft skills, as well as technical skills related to the assignment.
\end{abstract}

Keywords: soft skills, doccumentary, undergraduate, motivation, ethical responsibility, survey

\begin{abstract}
Resumen
El desarrollo de documentales amateur tipo screencast y su autoevaluación, coevaluación y evaluación por pares ha demostrado ser una herramienta formativa muy útil para el entrenamiento de la responsabilidad ética en el ámbito universitario de ciencias de la vida. Parte del éxito se debe sin duda al hecho de conseguir aprovechar la motivación del alumnado, que reconoce, por una parte, que se trata de un reto atractivo $y$, por otra, habérselo pasado bien en su desarrollo más allá del encargo académico. El uso de encuestas al alumnado ha permitido comprobar la valoración que éste hace de la repercusión de la actividad sobre el entrenamiento de componentes especificas de la responsabilidad ética. Del mismo modo, ha permitido comprobar los efectos positivos de la herramienta para entrenar otras competencias transversales, así como competencias técnicas relacionadas con el encargo.
\end{abstract}

Palabras clave: competencias transversales, documental, grado, motivación, responsabilidad ética, encuesta 
El screencast como herramienta para desarrollar responsabilidad ética y otras competencias transversales: percepción del alumnado

\section{Introducción}

La biotecnología ha experimentado un gran avance a partir del descubrimiento de la doble hélice a mediados del siglo XX. En este momento, se dispone de acceso a mucha información acerca de cada individuo a través del diagnóstico genético, y a nivel técnico es posible reproducir in vitro y reparar o incluso mejorar al individuo alterando su composición genética. Este avance plantea al mismo tiempo esperanzas y temores (Capella, 2015) y debe analizarse críticamente desde el punto de vista de la ética. Aún más, las tecnologías relacionadas con la edición de genes no solo plantean problemas de seguridad y riesgos y beneficios para la salud, sino que ponen en peligro principios éticos como la dignidad humana, la justicia, la equidad, la proporcionalidad y la autonomía (European Group on Ethics in Science and New Technologies, 2016). No solo es un problema con respecto a la tecnología en sí misma, sino también sobre cómo se implementa. Como ejemplo, en el dictamen $\mathrm{n}^{\circ} 29$ del European Group on Ethics in Science and New Technologies en 2015, se hace ver que cualquier individuo puede obtener fácilmente un análisis genómico completo, pero que las pruebas utilizadas por diferentes compañías han dado resultados variables, lo que obviamente genera preocupación (European Group on Ethics in Science and New Technologies, 2016).

Además de los problemas éticos que surgen de la tecnología específica y su uso, el comportamiento de los investigadores también implica problemas éticos. La alta competitividad en el sector de la investigación es evidente y el lema "publicar o perecer", ya empleado en 1932, se ha convertido en una dura realidad (Rawat y Meena, 2014). El estrés para desarrollar la mayor cantidad de publicaciones posible proporciona un caldo de cultivo para cuestiones éticas en la investigación. En consecuencia, las malas conductas de investigación se revelan, tristemente, de forma continua como ponen de relieve los trabajos de Errami y Garner (2008) y Bohannon (2013), entre otros.

En este contexto, la capacitación de responsabilidad ética en los investigadores debería ser cada vez más importante. Los estudios de pregrado generalmente se enfocan en el conocimiento básico requerido para adquirir las competencias técnicas específicas correspondientes a una carrera determinada, pero se pone poco énfasis en el desarrollo de competencias transversales, altamente valoradas por los empleadores. Entre ellas, las competencias relacionadas con la ética han sido frecuentemente ignoradas, aunque esta situación parece cambiar. De hecho, las entidades de acreditación como el Accreditation Board for Engineering and Technology, Inc. (ABET) han llamado la atención sobre la importancia de la ética en el currículo de ingeniería de grado. No obstante, la realidad es que la integración de la educación ética en los planes de estudios de ciencia e ingeniería sigue siendo un trabajo en progreso (Berry et al., 2013).

Los resultados relacionados con la ética son, por otra parte, bastante diferentes de los correspondientes a las ciencias básicas y la ingeniería. En un contexto en el que profesores y estudiantes están acostumbrados a estudiar y evaluar los resultados relacionados con el dominio cognitivo, es difícil integrar los resultados relacionados con el dominio afectivo, como es el caso de la ética (Hassel et al., 2015). De hecho, surgen nuevas alternativas para integrar enfoques humanistas en contextos científicos. Por ejemplo, con técnicas como la Midstrem Modulation (regulación de mitad de camino), donde un humanista se integra e interactúa con los 
investigadores para involucrarlos con los aspectos sociales y éticos de su trabajo (Flipse et al., 2013).

Mulhearn et al. (2017) en un extenso meta-análisis de metodologías docentes aplicadas a la adquisición de esta competencia observaron que los tipos de aproximación más habitual son la discusión general y adherencia a la norma. El primer tipo generalmente se aborda mediante discusiones en grupos de tamaño variable, mientras el segundo, más basado en moral filosófica, se aborda mediante clases magistrales. El tipo de enfoque empleado en la presente innovación docente, en la que el alumnado desarrolla un documental amateur analizando los aspectos éticos de una determinada tecnología, se enmarcaría entre las metodologías referidas por Mulheran et al. (2017) como autoreflexión filosófica y enseñanza basada en ejemplos. En la primera tienen cabida las reflexiones sobre dilemas éticos contemporáneos y se basa en mayor medida en el desarrollo de trabajos académicos escritos. La segunda es bastante parecida, pero se basa en el uso de ejemplos de situaciones éticas prominentes y se suele abordar mediante clases magistrales.

La iniciativa surgió hace varios años, cuando se evaluaron los estilos de aprendizaje de los estudiantes en el contexto de las Ciencias de la Vida y la Ingeniería en la Universitat Politècnica de València (UPV). Siguiendo el índice de Felder y Silverman (1988), se concluyó que estos estudiantes tendían a ser activos, sensoriales, visuales y secuenciales (Peiró et al., 2012). Parecía entonces que la producción de documentales amateur o screencasts podría representar una herramienta interesante para desarrollar habilidades transversales en estudiantes de grado.

En otros marcos docentes, los investigadores habían aplicado el uso de presentaciones orales sobre conflictos éticos como elemento iniciador de debates (Cebolla-Cornejo, 2009). Sin embargo, esta aproximación no se puede abordar en contextos docentes caracterizados por un bajo número de horas presenciales y un número elevado de alumnos. Por ello, surgió la alternativa de proponer a los alumnos realizar los mencionados screencasts. Esta metodología lleva varios años aplicándose en el grado de Biotecnología, en la que el alumnado desarrolla un documental evaluando aspectos éticos del trabajo como investigador en el ámbito de la Biotecnología y proponiendo un decálogo del buen investigador (Cebolla-Cornejo et al., 2017).

En el caso del grado de Ingeniería Biomédica de la UPV, también se ha empleado con éxito el uso de screencasts como metodología docente (Cebolla-Cornejo et al., 2016). En este caso, el alumnado debe preparar en grupo un documental amateur sobre los aspectos éticos relacionados con una determinada tecnología, normalmente relacionada con la biomedicina, en grupos de cinco estudiantes. Estos documentales posteriormente se coevalúan, evalúan por pares y autoevalúan, con el objetivo de promover la objetividad como una pieza clave adicional dentro de la responsabilidad ética.

\section{Objetivos}

Los beneficios del uso del screencast como herramienta para desarrollar la competencia de responsabilidad ética ya se han analizado en estudios previos. El objetivo del presente estudio consistió en recabar la opinión de los propios estudiantes sobre la utilidad de la actividad propuesta para desarrollar aspectos concretos de dicha competencia, así como comprobar si también percibían que a su vez estaban entrenando otras competencias transversales. 
El screencast como herramienta para desarrollar responsabilidad ética y otras competencias transversales: percepción del alumnado

\section{Desarrollo de la innovación}

\subsection{Contexto académico}

La investigación se desarrolló con estudiantes de tercer curso del Grado en Ingeniería Biomédica en el marco de la asignatura obligatoria Bioética y ética profesional (64 estudiantes en el curso académico 2017-2018) de la Universitat Politècnica de València.

\subsection{La actividad y su evaluación}

Se organizó a los alumnos en grupos de cinco personas a su propia elección y se pidió al grupo que preparara un documental amateur en forma de screencast de entre 15 y 20 minutos. El screencast es un vídeo en el que se parte de una interfaz gráfica que se ejecuta en la pantalla del ordenador y se graba la voz en off de un narrador. La interfaz gráfica puede ser una presentación tipo powerpoint o prezzi, animaciones o secuencias de vídeo. Alternativamente los alumnos pueden presentar situaciones teatralizadas como pequeños cortometrajes o entrevistas.

El screencast debía abordar cuestiones éticas relacionadas con la investigación: concretamente los estudiantes debían seleccionar una tecnología o grupo de tecnologías e identificar y reflexionar sobre los dilemas éticos que conlleva su utilización.

Una vez desarrollada la actividad (producción del screencast) se realizaron las correspondientes evaluaciones (profesor, coevaluación, autoevaluación y evaluación por pares). Finalmente se realizó un cuestionario en línea para analizar la percepción del alumno sobre las actividades propuestas. Este cuestionario estuvo configurado por doce cuestiones sobre la utilidad de la actividad para desarrollar la competencia de responsabilidad ética, nueve cuestiones sobre la utilidad de la actividad para desarrollar otras competencias transversales, cuatro cuestiones sobre la utilidad de la actividad para desarrollar competencias específicas de corte técnico, dos cuestiones sobre el reparto de actividades en el grupo y cinco cuestiones para analizar aspectos motivacionales. Todas las cuestiones se plantearon en escala tipo Likert de cinco puntos.

\section{Resultados y discusión}

Desde el punto de vista del profesor, los screencast contribuyen a entrenar la responsabilidad ética, como puso de manifiesto anteriormente el análisis de los resultados de las evaluaciones realizadas (Cebolla-Cornejo, 2016). Pero más allá de comprobar si los alumnos realmente eran capaces de identificar los problemas éticos que surgen con el uso de determinadas tecnologías, era importante conocer su opinión sobre si realmente la actividad había sido útil para desarrollar ésta y otras competencias transversales.

El pase de encuestas tras haber realizado la actividad, incluyendo el desarrollo del screencast y las diferentes evaluaciones, fue exitoso, obteniendo un nivel de respuesta del 92,2\%. De este modo, los resultados obtenidos se pueden considerar representativos de la población estudiada. 
La opinión del alumnado confirmó que efectivamente la herramienta había contribuido a desarrollar la competencia de responsabilidad ética, ya que el $84,8 \%$ estuvo de acuerdo o totalmente de acuerdo con esta afirmación (Fig. 1).

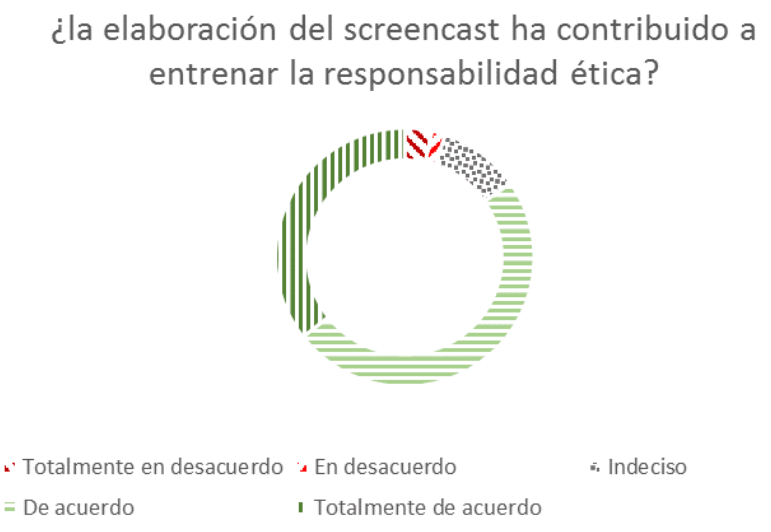

Fig. 1 Percepción del alumnado sobre el efecto de la actividad en el entrenamiento de la responsabilidad ética.

Esta competencia es especialmente compleja y cuenta con distintos aspectos o dimensiones a tener en cuenta. Por ello, con el objetivo de profundizar sobre qué componentes de la competencia se había actuado, se les preguntó sobre la utilidad de la actividad para entrenar distintos aspectos de la competencia. Se seleccionaron como punto de partida los niveles de dominio de la competencia seleccionados por la Universitat Politècnica de València dentro del proyecto de establecimiento de un sistema de medición de competencias transversales (http://www.upv.es/contenidos/COMPTRAN), que fueron modificados o completados según se creyó oportuno para facilitar que el alumno comprendiera los aspectos a los que se estaba haciendo referencia en el cuestionario. Así, de forma concreta, al alumnado se le preguntó hasta qué punto el desarrollo de la actividad había contribuido a entrenar los siguientes aspectos:

RE-1: Interpretar y valorar críticamente la información y la realidad

RE-2: Fundamentar y argumentar los juicios propios

RE-3: Tomar conciencia de otra manera de ver y percibir las cosas

RE-4: Aceptar críticamente nuevas perspectivas, aunque cuestionen las propias

RE-5: Identificar aspectos éticos en el uso de la tecnología

RE-6: Identificar los agentes afectados por una tecnología

RE-7: Reflexionar sobre las consecuencias y efectos de las tecnologías

RE-8: Aplicar los conceptos éticos y deontológicos de la profesión

RE-9: Proponer decisiones respecto al uso de la tecnología desde la reflexión de los principios éticos que se vean afectados

RE-10: No discriminar a las personas por razones de diferencia social, cultural o de género 
El screencast como herramienta para desarrollar responsabilidad ética y otras competencias transversales: percepción del alumnado

\section{RE-11: Tratar a los demás con objetividad}

De forma mayoritaria los alumnos opinaron que efectivamente la actividad les había ayudado a interpretar y valorar críticamente la realidad, con un $84,7 \%$ de alumnado conforme, fundamentar y argumentar os juicios propios, $91,5 \%$ de conformidad, tomar conciencia de otras maneras de ver y percibir las cosas, 75,8\% de conformidad (Fig. 2). Se mostraron menos contundentes a la hora de valorar el aspecto RE-4, aceptar críticamente nuevas perspectivas, aunque cuestionen las propias, con un nivel de conformidad del 69,5\%. Además, en este caso, también se observó un mayor grado de indecisión. Éste probablemente esté relacionado con el hecho de que en estos casos la tecnología evaluada no hubiese conllevado que tuvieran que replantearse su perspectiva de los problemas analizados.

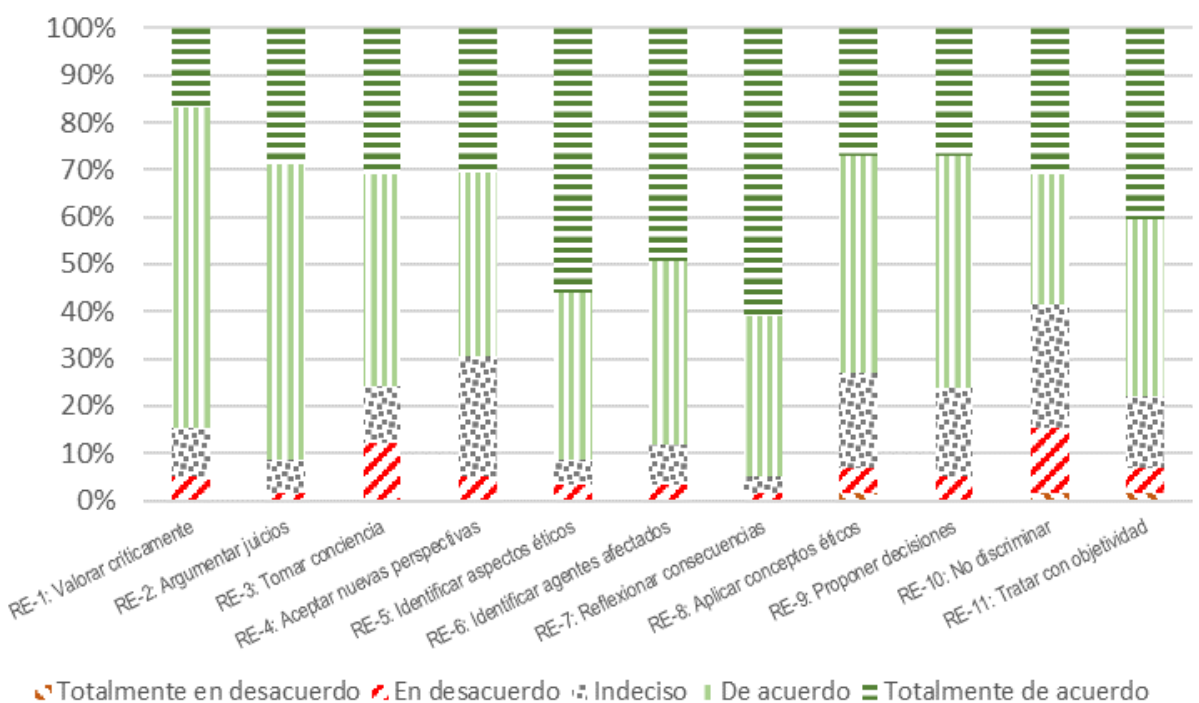

Fig. 2 Opinión del alumnado sobre la utilidad de la actividad para entrenar distintos aspectos relacionados con la responsabilidad ética.

Respecto a aspectos concretos de las tecnologías analizadas en su trabajo, el 91\% del alumnado estuvo de acuerdo o totalmente de acuerdo en la utilidad de la actividad para identificar los aspectos éticos del uso de la tecnología, identificando los agentes afectados por dicha tecnología $(88,2 \%)$ y reflexionando sobre las consecuencias y efectos de las mismas $(94,9 \%)$.

Aunque de forma mayoritaria, los estudiantes fueron menos terminantes fueron a la hora de valorar el aspecto RE-8, aplicar los conceptos éticos y deontológicos de la profesión, con un grado de conformidad del 72,9\% (Fig. 1). Aquí de nuevo apareció un mayor grado de incertidumbre (20,3\%), que quizá pudiese estar relacionado con el hecho de que no hubieran entendido bien la pregunta. Esto es así, puesto que dicho análisis es prácticamente un requisito previo para proponer decisiones respecto al uso de la tecnología desde la reflexión de los 
principios éticos que se vean afectados, RE-9, un aspecto con el que el 76,3\% estuvo de acuerdo en haber entrenado gracias a la actividad.

Aun siendo mayoritaria la opinión de acuerdo o totalmente de acuerdo $(58,7 \%)$ respecto a la contribución de la actividad a la hora de no discriminar a las personas por diferentes razones (RE-10), lo cierto es que el apoyo fue menor. De nuevo, el grado de indecisión fue elevado $(25,9 \%)$. Este hecho junto con el análisis de los trabajos entregados pone de relieve que en muchos casos las tecnologías analizadas no implicaban una clara discriminación, más allá del principio de justicia relacionado con el acceso a la tecnología, pero la discriminación por bases económicas no se contemplaba en la pregunta.

Finalmente, dentro de este bloque se preguntó si la actividad había contribuido a entrenar el hecho de tratar a los demás con objetividad. Más que en el screencast, el entrenamiento de este aspecto estuvo relacionado con las autoevaluaciones, coevaluaciones y evaluaciones por pares desarrolladas. En ellas los alumnos debían valorar el trabajo de su grupo de forma global, su propia contribución y la del resto de integrantes del grupo al trabajo (evaluación por pares) y el trabajo desarrollado por otros grupos (coevaluación). Se trataba de entrenar la objetividad como pieza clave de la responsabilidad ética, puesto que en el futuro se verán enfrentados a la necesidad de evaluar el trabajo o financiación de compañeros y subordinados. En este caso, el $78,0 \%$ del alumnado estuvo de acuerdo en que la actividad en su conjunto habría contribuido a entrenar esta componente de la competencia.

Experiencias anteriores ya anticiparon que el uso de screencasts permitía a los alumnos entrenar otras competencias transversales, más allá de la específica de la experiencia: la responsabilidad ética (Cebolla-Cornejo et al., 2016; Cebolla-Cornejo et al., 2017). Sin embargo, se decidió extender este análisis de forma que el fuera el propio alumnado quien confirmara si la actividad había contribuido a trabajar otras competencias.

El grado de conformidad fue mayoritario ( $>89 \%)$ para las competencias de búsqueda de información, pensamiento crítico y conocimiento de problemas contemporáneos (Fig. 3). Menor porcentaje de acuerdo se obtuvo para síntesis de información y comunicación oral, aunque por encima del 74\%. Estos resultados eran esperados, puesto que para el desarrollo del trabajo los alumnos deben buscar información, analizarla críticamente, sintetizarla y comunicarla de forma oral. Por otro lado, el análisis de la información les llevaría necesariamente a conocer problemas contemporáneos relacionados con el uso de una tecnología específica.

Fue especialmente destacable el grado de conformidad sobre la utilidad para desarrollar la creatividad, ya que el 94,9\% estuvo conforme en que la actividad había contribuido a entrenar esta competencia y entre ellos el 66,1\% estuvo totalmente de acuerdo (Fig. 3). Es cierto que la originalidad era un aspecto importante a la hora de valorar el documental presentado, pero como se verá más adelante el énfasis puesto en desarrollar la creatividad también pudo estar relacionado con aspectos motivacionales.

La actividad propuesta no deja de ser un trabajo de equipo, por lo que no es de extrañar que un 93,1\% estuviera de acuerdo o totalmente de acuerdo en la utilidad para trabajar esta competencia (Fig. 3). Menor grado de conformidad se obtuvo al preguntar sobre el efecto en la competencia de liderazgo, algo lógico puesto que es raro que en los equipos formados todos los miembros 
El screencast como herramienta para desarrollar responsabilidad ética y otras competencias transversales: percepción del alumnado

hubieran asumido una posición de liderazgo. De hecho, en las evaluaciones por pares, se pudo comprobar que en muchos grupos se dio una aportación especialmente relevante de uno o dos miembros. Aunque estos datos no se muestran por exceder el ámbito del presente trabajo.

Respecto a la planificación del tiempo, el 69,0\% estuvo de acuerdo o totalmente de acuerdo en que había trabajado la competencia en el desarrollo de la actividad. Análisis previos con la misma actividad en años anteriores con el mismo contexto académico (Cebolla-Cornejo et al., 2016) pusieron de relieve que el tiempo dedicado a la actividad seguía una distribución de Poisson con una asimetría negativa (hacia el menor número de horas dedicadas), con una media de 9,15 de horas dedicadas a la preparación del trabajo y una mediana de 8 horas.

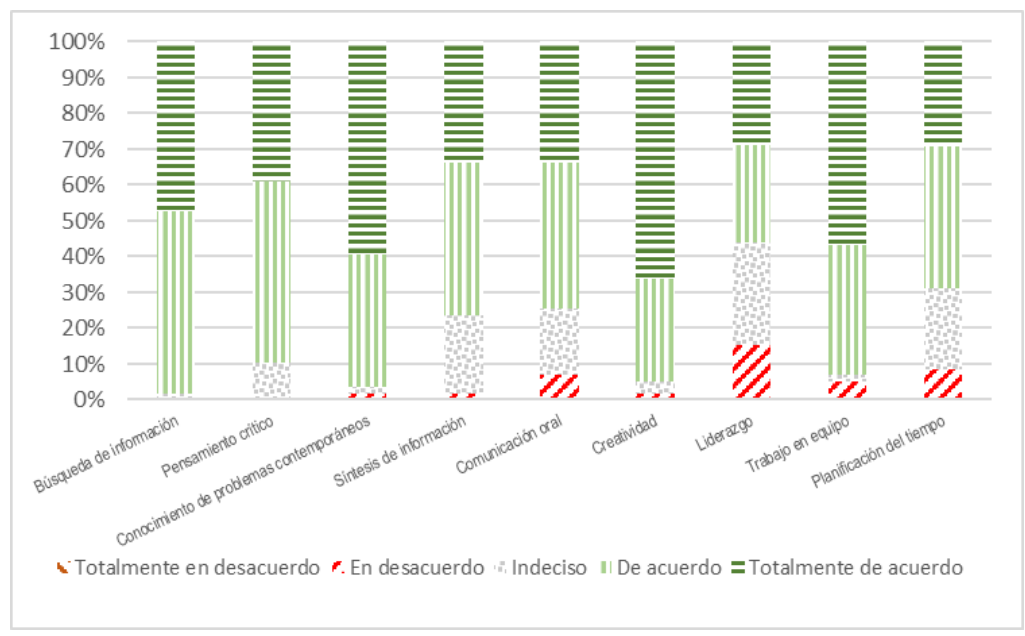

Fig. 3 Opinión del alumnado sobre la utilidad de la actividad para entrenar otras competencias transversales.

Más allá de competencias transversales generales, se pidió opinión sobre si la actividad había permitido entrenar competencias más específicas y de corte técnico relacionadas con el encargo (Fig. 4). Efectivamente, alrededor del $80 \%$ estuvo de acuerdo o totalmente de acuerdo en que les había servido para trabajar aspectos relacionados con el diseño gráfico, el manejo de software de edición de vídeo e interfaz gráfico para presentaciones y la elaboración de guiones. 


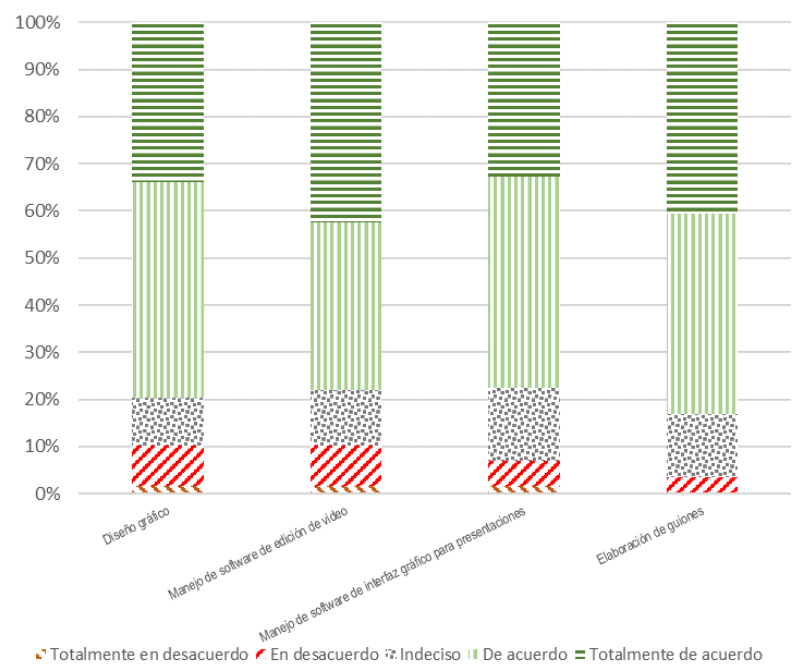

Fig. 4. Opinión del alumnado sobre la utilidad de la actividad para entrenar competencias específicas relacionadas con aspectos técnicos.

En este punto cabe destacar que casi la mitad del alumnado reconoció haber sido ayudado por otros miembros del grupo para desarrollar la parte gráfica de su contribución (Fig. 5). Aunque en muchos casos pudo estar relacionado por el hecho de que algo más de la mitad del alumnado reconoció que uno o dos miembros del grupo se había encargado específicamente del desarrollo de la parte gráfica $(61,0 \%)$.
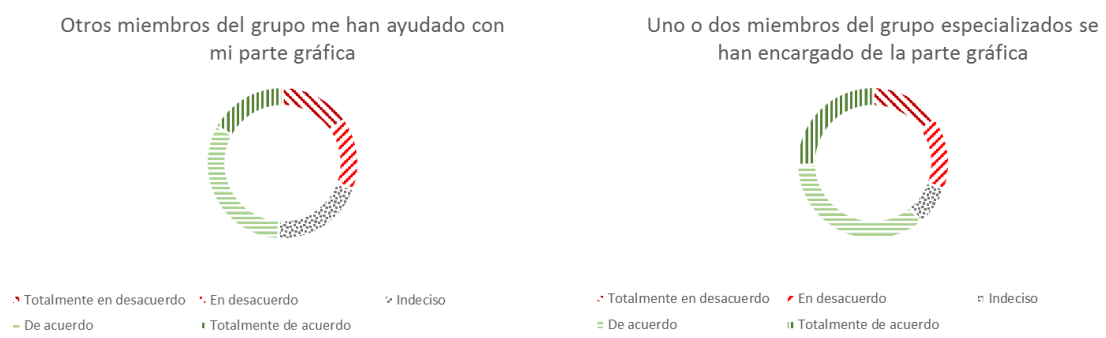

Fig. 5. Opinión del alumnado sobre el desarrollo de la parte gráfica de la actividad.

Finalmente se preguntó al alumnado sobre aspectos de tipo motivacional. Casi el 75\% estuvo conforme en que se encontraba más cómodo con entornos digitales (Fig. 6). Un resultado esperado en una generación que ya puede ser considerada digital. De hecho, estudios de más de diez años de antigüedad ya ponían de relieve la naturalidad con la que los estudiantes se desenvuelven en entornos digitales. Así, se comprobó que los estudiantes constituyen el grupo de usuarios más grande en cuestión de sesiones iniciadas y páginas visitadas y era más probable que sus sesiones fueran más largas al estudiar el uso de recursos digitales académicos en el ámbito universitario (Nicholas et al., 2008). 
El screencast como herramienta para desarrollar responsabilidad ética y otras competencias transversales: percepción del alumnado

Sin embargo, en contra de lo que se esperaba, los estudiantes se dividieron entre los que estaban más cómodos en un entorno video que un texto, el 35\%, y los que preferían el último, el 32,2\% (Fig. 6). Este resultado es inusual en tanto que, al menos en educación en línea, a distancia y a nivel de máster, se ha comprobado que el medio vídeo mejora la capacidad de retención y la motivación de los estudiantes (Choi y Johnson, 2005). No obstante, también es cierto que la permanencia, facilidad de distribución y familiaridad del texto hace que las ideas y comentarios del alumnado y profesorado sean más fácil y convenientemente accesibles (Hill et al., 2009).

En cualquier caso, al ser preguntados de forma más precisa sobre si preferirían haber realizado un trabajo escrito o el screencast, el 54,2\% prefirió el entorno vídeo frente al $25,2 \%$ que prefería el entorno texto (Fig. 6). Hay que resaltar en este punto que este era el primer documental amateur al que se enfrentaban como encargo académico los estudiantes.

Más allá de la preferencia y comodidad del alumnado por trabajar en entornos de vídeo o texto, quedó clara la vertiente motivacional de la actividad propuesta, ya que el 83,0\% del alumnado estuvo conforme con que preparar el documental había representado un reto atractivo y el $69,5 \%$ reconoció que más allá del encargo académico se lo había pasado bien preparando el screencast (Fig. 6).

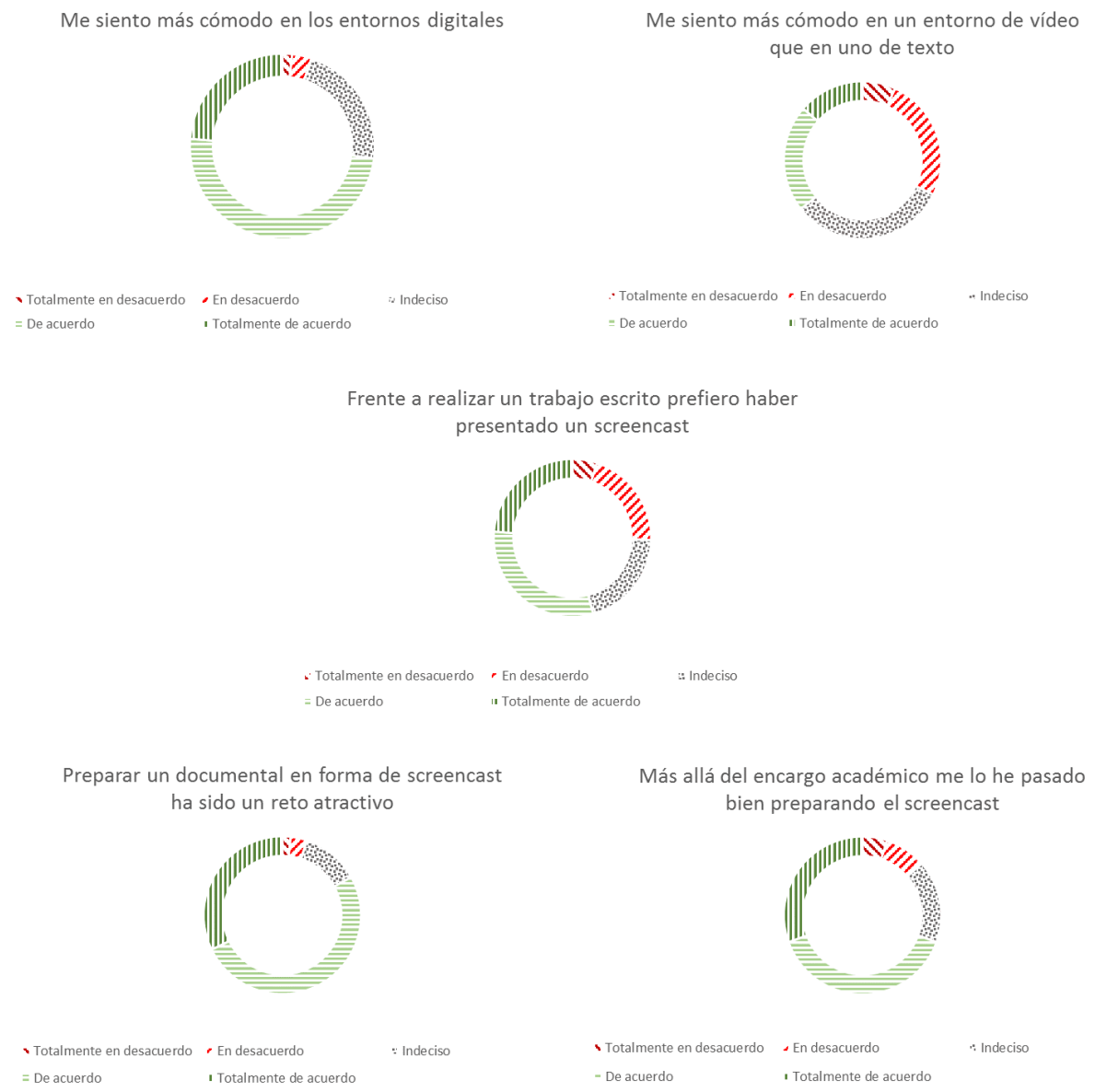

Fig. 6. Opinión del alumnado sobre aspectos de tipo motivacional. 


\section{Conclusiones}

El desarrollo de documentales amateur tipo screencast para reflexionar sobre los aspectos éticos de una tecnología específica representa una herramienta muy útil para entrenar la competencia de responsabilidad ética, a tenor de la propia opinión del alumnado. En parte, puede explicarse por una componente motivacional, ya que a pesar de que los estudiantes reconocieron sentirse igual de cómodos en entornos de vídeo que de texto, lo cierto es que consideraron que preferían esta metodología frente a un trabajo escrito y reconocieron que realizar un documental amateur representó un reto atractivo. De hecho, mayoritariamente opinaron que, más allá del encargo académico, se lo habían pasado bien.

La metodología permitió desarrollar la competencia de responsabilidad ética forma global, actuando sobre el entrenamiento de sus distintas componentes. Es cierto que las componentes más relacionadas específicamente con el encargo, como identificar aspectos éticos, agentes afectados y consecuencias de una tecnología, recibieron mayor grado de apoyo en cuanto la contribución del trabajo a su entrenamiento. Pero también reconocieron que otras más tangenciales también fueron entrenadas, incluyendo, valorar críticamente, argumentar juicios, tomar conciencia de otras formas de ver las cosas, aceptar nuevas perspectivas, aplicar conceptos éticos y proponer decisiones. El tema del trabajo, es cierto que tuvo menos peso a la hora de entrenar la no discriminación, en cuanto que las tecnologías evaluadas por los estudiantes no conllevaban per se discriminación, más allá de las posibilidades de acceso a la misma. Por otro lado, las autoevaluaciones, coevaluaciones y evaluaciones por pares, sin duda, contribuyeron a entrenar la objetividad.

Finalmente, los estudiantes reconocieron que la metodología propuesta contribuyó a formar en otras competencias. Especialmente la creatividad, aunque también competencias como búsqueda de información, pensamiento crítico, conocimiento de problemas contemporáneos, síntesis de información, comunicación oral y planificación del tiempo. Al tratarse de un trabajo grupal, también reconocieron haberse formado en la competencia de trabajo en grupo y en menor medida en liderazgo, probablemente porque sólo uno o dos miembros por grupo parecieron asumir ese rol. Por otro lado, también contribuyó a formar competencias de tipo técnico como el diseño gráfico, edición de vídeo, interfaces gráficas y elaboración de guiones.

\section{Agradecimientos}

La presente investigación no ha recibido financiación directa para su desarrollo y difusión. Los autores agradecen la colaboración del alumnado.

\section{Referencias}

BERRY, R.M., BORENSTEIN, J., BUTERA, R.J. (2013) "Contentious problems in bioscience and biotechnology: A pilot study of an approach to ethics education", Science and Engineering Ethics, 19(2), pp.653-668. 
El screencast como herramienta para desarrollar responsabilidad ética y otras competencias transversales: percepción del alumnado

BOHANNON, J. (2013) “Who’s afraid of peer review“. Science, 342, pp. 60-65.

CAPELLA, V.B. (2015) "Biotechnology, Ethics, and Society: The Case of Genetic Manipulation" en Gonzalez, W. New Perspectives on Technology, Values, and Ethics, Springer International Publishing, pp. 123-143.

CEBOLLA-CORNEJO, J. 2009. "Metodología aplicada a la formación en valores en entornos docentes de elevada carga técnica y científica", Jornadas de innovación: Metodologías activas para la formación en competencias y estrategias de evaluación alternativas, Instituto de Ciencias de la Educación UPV, pp. 1-3.

CEBOlla-CORNEJO, J., LEIVA-BRONDO, M., PEIRÓ, R., PÉREZ-DE-CASTRO, A.M. (2016) "Screencasts as a tool for developing transferable skills in undergraduate students: analysis of an evaluation experience", INTED2016 Proceedings, pp. 2360-2365.

CEBOlla-CORNEJO, J., LEIVA-BRONDO, M., PEIRÓ, R., PÉREZ-DE-CASTRO, A.M. (2017) "Training ethical responsibility as a transferable skill in undergraduate students of Biotechnology”, INTED2017 Proceedings, pp. 6772-6778.

CHOI, H.J., JOHNSON, S.D. (2005) "The effect of context-based video instruction on learning and motivation in online courses". The American Journal of Distance Education, 19(4), pp. $215-$ 227.

ERRAMI, M., GARNER, H. (2008) “A tale of two citations”, Nature, 451, pp.397-399.

EUROPEAN GROUP ON ETHICS IN SCIENCE AND NEW TECHNOLOGIES (2015) “The ethical implications of new health technologies and citizen participation", Publications Office of the European Union, Luxemburg, 86 pp.

EUROPEAN GROUP ON ETHICS IN SCIENCE AND NEW TECHNOLOGIES (2016) "Statement on Gene Editing", Publications Office of the European Union, Luxemburg, 2 pp.

FELDER, R.M., SILVERMAN, L.K. (1988) "Learning and teaching styles in engineering education”, Engineering Education, 78(7), pp. 674-681.

FLIPSE., S.M., VAN DER SANDEN, M.C., OSSEWEIJER, P. (2013) "Midstream modulation in biotechnology industry: Redefining what is 'part of the job'of researchers in industry", Science and Engineering Ethics, 19(3), pp. 1141-1164.

HASSEL, K., QUINLAN, P., MORESOLI, C. (2015) "Impacts and ethics of biotechnology as a vehicle for assessing CEAB outcomes in a first year chemical engineering course at the University of Waterloo". Proceedings of the Canadian Engineering Education Association, june 2015, Hamilton, Ontario, Canada.

HILL, J.R., SONG, L., WEST, R.E. (2009) "Social learning theory and web-based learning environments: A review of research and discussion of implications". The American Journal of Distance Education, 23(2), pp. 88-103.

MULHEARN, T.J., STEELE, L.M., WATTS, L.L., MEDEIROS, K.E., MUMFORD, M.D., CONNELLY, S. (2017). "Review of Instructional Approaches in Ethics Education". Science and Engineering Ethics, 23, pp. 883-912. 
NICHOLAS, D., HUNTINGTON, P., JAMALI, H.R., ROWLANDS, I. AND FIELDHOUSE, M. (2009) "Student digital information-seeking behaviour in context" Journal of Documentation, 65(1), pp. 106-132.

PEIRÓ, R.M., PÉREZ-DE-CASTRO, A.M., ESTERAS, C., MERLE, H. , FERRIOL, M., DÍEZ, M.J., PICÓ, B., LISÓN, P., LÓPEZ-GRESA, M.P., CEBOLLA-CORNEJO, J. , LEIVABRONDO, M. (2012) "Estilos de aprendizaje para el diseño de materiales docentes de estudiantes universitarios en el área de ciencias de la vida", IX foro internacional sobre la evaluación de la calidad de la investigación y de la educación superior (FECIES). Santiago de Compostela (España), pp. 715-720.

RAWAT, S., MEENA, S. (2014) "Publish or perish: Where are we heading?", Journal ofResearch in Medical Sciences, 19(2), p.87. 